\title{
Effect of Sodium Hyaluronate on Inflammatory Cytokine Levels in Synovial Fluid of Patients with Knee Osteoarthritis
}

\author{
H. QU, F. WANG ${ }^{1}$, Z. LI, X. LI ${ }^{2}$, Z. LI ${ }^{3}$, H. WU, FAN WU AND L. SHI ${ }^{2 *}$
}

Department of Orthopaedics, Hubei Provincial Hospital of Integrated Chinese and Western Medicine, Hubei, ${ }^{1}$ Department of Spine, Characteristic Medical Centre of the Chinese People's Armed Police Force, ${ }^{2}$ Department of Orthopaedics, Yuyao Traditional Chinese Medicine Hospital of Zhejiang Province, Zhejiang, ${ }^{3}$ Central War Zone General Hospital of the Chinese People's Liberation Army, P. R. China

Qu et al.: Sodium Hyaluronate and Inflammatory Cytokine Levels in Synovial Fluid

\begin{abstract}
This study evaluated the efficacy of intraarticular injection of sodium hyaluronate in the treatment of knee osteoarthritis and its influence on the levels of stromal cell-derived factor-1 and matrix metalloproteinases 3,9 and 13 in the synovial fluid of patients with knee osteoarthritis. A total of 180 patients with knee osteoarthritis treated in the orthopaedic outpatient department of the Hubei Provincial Hospital from April 2015 to April 2017 were selected as the research objects and all of them were given intraarticular injection of sodium hyaluronate with synovial fluid extracted before the injection, 1 and 3 mo after the injection, and the level of stromal cell-derived factor- 1 and matrix metalloproteinases 3, 9, 13 were determined using a sandwich enzyme-linked immunosorbent assay method. After 3 mo of treatment, the effect on the efficacy of patient's gender, age, body mass index, swollen joint degree, involved joint space, Kellgren and Lawrence grading as well as tibiofemoral angle were observed and studied. Among the 180 cases there were 132 cases with $73.3 \%$ effective rate and 48 cases of with ineffective rate of $26.7 \%$. Significant differences were found in the levels of stromal cell-derived factor-1, matrix metalloproteinases 3,9 and 13 in the synovial fluid before the treatment, 1 and 3 mo after the treatment $(p<0.05)$ and the change of stromal cell-derived factor- 1 was positively correlated with that of matrix metalloproteinases 3,9 and 13 $(r=0.462, p<0.05 ; r=0.407, p<0.05 ; r=0.378, p<0.05$, respectively $)$. Multivariate analysis showed that the influential factors included the joint graded more than II, knee osteoarthritis involving tibiofemoral joint space and X-ray Kellgren and Lawrence grading level more than 3 with $R R>1.7$ in joint effusion and osteoarthritis involving tibiofemoral joint gap. In the treatment of knee osteoarthritis, the intraarticular injection of sodium hyaluronate could effectively reduce the levels of stromal cell-derived factor-1, matrix metalloproteinases 3,9 and 13 in synovial fluid and decrease inflammatory reaction. But it should be used with great caution for treating patients with moderate and severe knee osteoarthritis involving tibial and femoral joints with the joint effusion graded above II or bone contusion.
\end{abstract}

Key words: Stromal cell derived factor-1, osteoarthritis, sodium hyaluronate, matrix metalloproteinase 3, matrix metalloproteinase 9, matrix metalloproteinase 13

Osteoarthritis (OA) is a common disease in the middleaged and elderly mainly characterized by cartilage and subchondral bone destruction, seriously affecting the quality of life in patients and increases the medical expenditure of the government as well as the masses ${ }^{[1]}$. The incidence of OA of the knee joint, the main weightbearing joint as one of predilection sites is as high as $50 \%$ in people aged over $60^{[2]}$. Knee OA is one of the most common diseases with serious symptoms like pain and stiffness. There is no specific treatment to prevent the progression of OA. Sodium hyaluronate (HA) is a linear glycosaminoglycan composed of alternating disaccharide unit containing N2,N-acetylglucosamine and D2 glucuronic acid and it is one of the major components of articular cartilage and synovial fluid with the chemical nature of glycosaminoglycan. Intraarticular injection of HA is a method commonly used in clinical treatment, but it is strongly recommend against in the OA guidelines issued by American Academy of Department of Orthopaedics, Orthopaedic Surgeons (AAOS) in 2013. At present, there exists support, opposition as well as uncertainty in the matter 
of intraarticular injection of HA and there are many different guidelines proposed by various organizations, which makes clinicians puzzled whether to choose the injection ${ }^{[3-5]}$. Knee OA has different clinical manifestations such as pain, swelling, stiffness, friction and limited motion with varying severity. It is divided as patellofemoral joint, medial tibial femoral joint and lateral tibial femoral joint according to the involved sites. The main focus of this research is whether there is difference in the study results caused by the heterogeneity in OA cases.

HA and cartilage in the knee joint synovia play an important role in the development of OA and recent studies showed that the level of stromal cell derived factor-1 (SDF-1) and matrix metalloproteinases 3, 9 and 13 (MMP-3, 9 and 13) exert certain effects on cartilage degeneration of $\mathrm{OA}^{[6]}$. Currently there are few reports on the effect of HA injection on levels of inflammatory factors in joint fluid of OA patients. This investigation attempted to study the efficacy of intraarticular injection of HA for treating knee OA and to explore its effects on the level of SDF-1 and MMP-3, 9 and 13 in the synovial fluid, thus expecting to provide evidence for treating knee OA by intraarticular injection of HA.

A total of 180 patients with knee OA treated in the Hubei Provincial Hospital Orthopaedic Outpatient Department from April 2015 to April 2017 were enrolled in this study and the patients included 80 males and 100 females aged 40-75 with an average age of $58.5 \pm 1.7 \mathrm{y}$ and a disease course of $1-10 \mathrm{y}$, (average $6.3 \pm 1.2 \mathrm{y})$.

Patients were collected according to the American Rheumatic Association 1987 revised criteria for $\mathrm{OA}^{[5]}$. Inclusion criteria were, all collected MG patients were diagnosed with standard; patients with joint effusion; patients who signed the informed consent form. Exclusive criteria were patients with liver and kidney diseases, connective tissue diseases, endocrine diseases, severe cardiovascular diseases or cancer.

All patients were treated with intraarticular injection of HA, with inferior medial or lateral to patella as the puncture point, the injection was given using a $5 \mathrm{ml}$ syringe into the joint cavity with a sense of breaking through and no resistance to drug infusion. Patients were injected $2.5 \mathrm{ml}$ of HA followed by moving the knee several times to help the drug fully make contact with articular surface. The joint effusion, if any, was first removed followed by the HA injection. The course was once a $\mathrm{w}$ for $5 \mathrm{w}$.
All subjects were given conventional disinfection and took a sitting position at 90 degree of flexion, a disposable 5-ml syringe was used to puncture from infrapatellar medial or lateral approach into the joint cavity to extract all the synovial fluid without back streaming of blood, then the fluid samples were randomly numbered and divided into samples before injection, 1 mo after injection and 3 mo after injection. The selected sample was centrifuged with the supernatant stored at -80 degrees until assayed to detect levels of SDF-1 and MMP-3, 9 and 13 with sandwich ELISA using a commercial ELISA kit following the instructions provided with the kit.

Subjective symptoms were recorded to evaluate the efficacy in patients treated by HA injection for 3 mo with the treatment either effective or ineffective. Effective treatment described that the symptoms of pain, swelling, stiffness and disease activity were improved and or else, the treatment would be ineffective. All data were processed using SPSS 21 software. The general demographic characteristics and clinical features of the subjects were analysed by the descriptive and inferential statistics. Test of normality and homogeneity test of variance were conducted on the measurement data of age, body mass index (BMI) and tibiofemoral angle. According to the subjective evaluation of effect the patients were divided as effective group and ineffective group with a comparison of data in between. The measurement data accorded with normal distribution and homogeneity of variance were described as mean \pm standard deviation and compared by t-test, or by Mann-Whitney $U$ test. In addition patients were divided into 2 groups based on their age, 40 to $64 \mathrm{y}$ group and 65 to 75 y group and based on BMI divided as under $25 \mathrm{~kg} / \mathrm{m}^{2}$ and over $25 \mathrm{~kg} / \mathrm{m}^{2}$ followed by chi square test. The count data like gender, the involved joint space, swelling grade and Kellgren and Lawrence grading (K-L grading) were assessed using the chi square test after being appropriately grouped. The factors of statistical significance were believed to affect the efficacy of HA treatment for knee OA, and the relative risk (RR) as well as $95 \%$ confidence interval (CI) were calculated. The levels of SDF-1 and MMP-3, 9 and 13 in synovial fluid were analysed using Pearson correlation and hypothesis was assessed by a twosided test, $\mathrm{p}<0.05$ suggested that there was statistically significant difference.

Among the 180 selected cases there were 132 cases of effective treatment with an effective rate of $73.3 \%$ and 48 cases of ineffective treatment with a rate of $26.7 \%$. 
Significant differences were found among patients in levels of SDF-1, MMP-3, MMP-9 and MMP-13 in the synovial fluid between before treatment, 1 and 3 mo after the treatment $(\mathrm{p}<0.05)$, as shown in Table 1 . The correlation analysis of SDF-1, MMP-3, MMP-9 and MMP-13 levels before and after treatment showed that the change of SDF-1 was positively correlated with that of MMP-3, MMP-9 and MMP-13 ( $\mathrm{r}=0.462, \mathrm{p}<0.05$; $\mathrm{r}=0.407, \mathrm{p}<0.05 ; \mathrm{r}=0.378, \mathrm{p}<0.05)$.

According to the evaluation of subjective symptoms the patients were divided as effective group and ineffective group with no significant difference attributable to gender, unilateral or bilateral knee joint involvement and FTA ( $>0.05)$, while there was significant difference found with age, BMI, swelling degree, involved joint space and $\mathrm{X}$-ray $\mathrm{K}-\mathrm{L}$ grading $(\mathrm{p}<0.05)$, as shown in Table 2.

The indices of statistical difference were divided into 2 groups according to corresponding level, the exposed group and the non-exposed group, then the corresponding RR and $95 \%$ CI were calculated (Table 3 ). Among them the age was divided as $40-64 \mathrm{y}$ and $65-75 \mathrm{y}$; BMI under $25 \mathrm{~kg} / \mathrm{m}^{2}$ and over $25 \mathrm{~kg} / \mathrm{m}^{2}$; joint swelling grade I and grade over II; the involved joint space merely in patellofemoral joint and in tibiofemoral joint; and K-L grading was divided as the grade under 2 and the grade over 3 . There was no significant difference in age and BMI between groups. The influential factors included the joint graded over II, knee OA involving tibiofemoral joint space and X-ray

TABLE 1: INFLAMMATORY FACTOR LEVELS IN SYNOVIAL FLUID OF PATIENTS

\begin{tabular}{lccccc}
\hline Index & Before treatment & 1 month after treatment & 3 months after treatment & $\mathrm{F}$ & $\mathrm{P}$ \\
\hline SDF-1 & $723.6 \pm 45.5$ & $653.2 \pm 32.7$ & $428.1 \pm 24.6$ & 16.371 & $<0.05$ \\
MMP-3 & $190.4 \pm 32.6$ & $171.5 \pm 24.8$ & $146.2 \pm 13.9$ & 13.425 & $<0.05$ \\
MMP-9 & $436.3 \pm 27.8$ & $371.3 \pm 21.5$ & $305.6 \pm 19.8$ & 12.094 & $<0.05$ \\
MMP-13 & $512.7 \pm 51.4$ & $446.7 \pm 34.5$ & $386.3 \pm 29.5$ & 15.133 & $<0.05$ \\
\hline
\end{tabular}

TABLE 2: COMPARISON OF GENERAL DATA OF PATIENTS BETWEEN EFFECTIVE GROUP AND INEFFECTIVE GROUP

\begin{tabular}{|c|c|c|c|c|}
\hline Variable & Effective group $(n=132)$ & Ineffective group $(n=48)$ & $\mathrm{X}^{2}$ & $\mathrm{p}$ \\
\hline Age $(y)$ & & & 6.284 & $<0.05$ \\
\hline $40-64$ & 38 & 17 & & \\
\hline $65-75$ & 94 & 31 & & \\
\hline Gender & & & 0.982 & $>0.05$ \\
\hline Male & 50 & 30 & & \\
\hline Female & 82 & 18 & & \\
\hline BMI & & & 7.146 & $<0.05$ \\
\hline$\leq 25 \mathrm{~kg} / \mathrm{m}^{2}$ & 46 & 20 & & \\
\hline$>25 \mathrm{~kg} / \mathrm{m}^{2}$ & 86 & 28 & & \\
\hline Involved knees & & & 0.873 & $>0.05$ \\
\hline One knee & 70 & 32 & & \\
\hline Two knees & 62 & 16 & & \\
\hline Grading of knee swelling & & & 5.846 & $<0.05$ \\
\hline$|+| \mid$ & 82 & 42 & & \\
\hline III+IV & 50 & 6 & & \\
\hline Involved joint space & & & 12.651 & $<0.05$ \\
\hline Patellofemoral joint & 79 & 20 & & \\
\hline Medial tibiofibular joint & 12 & 12 & & \\
\hline Lateral tibiofibular joint & 11 & 6 & & \\
\hline Total joint & 30 & 10 & & \\
\hline X-ray K-L grading & & & 8.365 & $<0.05$ \\
\hline Grade 1 plus 2 & 90 & 34 & & \\
\hline Grade 3 plus 4 & 42 & 14 & & \\
\hline Average FTA $\left({ }^{\circ}\right)$ & $176 \pm 34$ & $175 \pm 36$ & 0.416 & $>0.05$ \\
\hline
\end{tabular}


TABLE 3: POTENTIAL RISK FACTORS AFFECTING HA TREATMENT OF KNEE OA

\begin{tabular}{lcc}
\hline $\begin{array}{l}\text { Potential influential } \\
\text { factors }\end{array}$ & RR $(95 \% \mathrm{Cl})$ & $\mathrm{p}$ \\
\hline Age $\geq 65 \mathrm{y}$ & $1.057(0.932,1.476)$ & 0.348 \\
$\mathrm{BMI} \geq 25 \mathrm{~kg} / \mathrm{m}^{2}$ & $1.071(0.953,1.342)$ & 0.415 \\
Joint effusion $\geq$ grade II & $1.752(1.168,2.575)$ & 0.016 \\
OA involved femorotibial & $1.822(1.341,2.608)$ & 0.001 \\
joint space & $1.563(1.107,2.342)$ & 0.005 \\
\hline
\end{tabular}

K-L grade over 3 with RR over 1.7 in joint effusion and OA involving tibiofemoral joint gap.

Multivariate analysis showed that the influential factors included the joint graded more than II, knee OA involving tibiofemoral joint space and X-ray K-L level more than 3 with $R R>1.7$ in joint effusion and OA involving tibiofemoral joint gap. All these suggest that in the treatment of knee OA with intraarticular injection of HA could effectively reduce the level of SDF-1, MMP-3, MMP-9 and MMP-1 in synovial fluid and inhibit inflammatory reaction, but it should be used with great caution for treatment of patients with moderate and severe knee OA involving tibial and femoral joint, the joint effusion graded above II or bone contusion.

HA has been widely applied in clinical practices with good results since it was first adopted by Peyron to treat OA in 1974. Knee intraarticular injection of HA can increase the content of HA in synovial fluid and enhance the viscoelasticity of articular fluid providing joint protection, lubrication, antiinflammation, pain control and repair of articular cartilage ${ }^{[7]}$. In 2013, the AAOS guideline strongly recommended against the HA intra-articular injection, which was merely considered to be of uncertainty in the first edition with the minimum clinical important improvement as an evaluating indicator. After the publication of the guideline, there are also many queries. Currently, the American Rheumatology Association and the OA research society international are still uncertain of HA treatment ${ }^{[8]}$. There existed disagreements in this issue among the top international organizations, which required clinicians to cautiously choose treatment methods. Actually uncertainty prevailed in clinical practice regarding the efficacy of HA treatment of knee $\mathrm{OA}$. It is difficult to determine which type of knee OA could be well treated by HA and there is no definite indication ${ }^{[9-10]}$. The purpose of this study is to explore the choice of HA indications. The results of this study showed that among the 180 selected cases there were 132 cases with an effective rate of $73.3 \%$ and 48 cases with ineffective rate of $26.7 \%$. Multivariate analysis showed that the influential factors included the joint graded more than II, knee OA involving tibiofemoral joint space and X-ray K-L level more than 3 with RR $>1.7$ in joint effusion and $\mathrm{OA}$ involving tibiofemoral joint gap. All these suggested that the intraarticular injection of HA should be used with great caution for treatment of patients with moderate and severe knee OA involving tibial and femoral joints, the joint effusion graded above II or bone contusion.

SDF-1 was originally discovered as a chemotactic factor released by bone marrow stromal cells and it has been recently shown that SDF-1 plays a key role in the pathological process of cartilage degeneration in patients with $\mathrm{OA}^{[11]}$. Synoviocytes in the joint can synthesize and secrete SDF-1 in synovial fluid and it, if combining with CXCR4 receptor in the surface of cartilage cell, can activate the signalling pathways of extracellular signal-regulated kinase (Erk) and associated kinase (p38MAP kinase) thereby inducing cartilage cells to release MMP-3, 9 and 13, all of which could cause damages to articular cartilage. Relevant data showed that MMP-3 is indirectly involved in degradation of type II collagen and it interacted with the corresponding inhibition factor to achieve the balance of cartilage metabolism ${ }^{[12]}$. When the balance is broken, the level of MMP is elevated, thus giving rise to cartilage degradation. Joint fluid contains a large amount of HA and provides liquid environment for the joint, which can not only lubricate joints, also nourish cartilage cells and remove cell waste and the product of extracellular matrix degradation. HA appears to be of macromolecular network structure in the joint fluid and it can regulate the diffusion of water as well as macromolecules and protect cartilage and synovial membrane from the invasion of bacteria, toxins and other immune complexes ${ }^{[13]}$. According to the results of this study, the levels of SDF-1 and MMP-3, 9, 13 were significantly decreased with the prolonging of time after the injection of HA, suggesting that the mechanism of HA in the treatment of knee OA is possibly to decrease the content of SDF-1 in synovial fluid and interfere its combination with CXCR4 receptor in chondrocytes so as to affect the release of MMP-3, 9 and 13; the injection of HA can supplement or improve its content in synovial fluid, increase its viscosity and lubrication of joint fluid, prevent inflammatory mediators or cartilage matrix degrading enzymes making contact with cartilage to avoid further damages of cartilage matrix; HA can reduce the number of inflammatory cells in the joints, inhibit or lower the secretion, release 
and diffusion of those enzymes and free radicals related to inflammatory reaction and decrease the permeability of synovial membrane and joint effusions; it can also inhibit the generation of pain-induced peptides, cover and protect pain receptors, inhibit the excitability of pain receptors and fibers in and under synovial membrane with the pain relief in joint; in addition HA can inhibit cartilage damage, accelerate its metabolism and repair articular cartilage ${ }^{[14,15]}$. Correlation analysis showed that SDF-1 was positively correlated with the levels of MMP-3, MMP-9 and MMP-13 ( $\mathrm{r}=0.462, \mathrm{p}<0.05$; $\mathrm{r}=0.407, \mathrm{p}<0.05 ; \mathrm{r}=0.378, \mathrm{p}<0.05)$. It is suggested that in the synovial fluid of patients with OA the contents of MMP-3, MMP-9 and MMP-13 increased with the increase of SDF-1 and also decreased with the lowering of SDF-1 content. This indicated that HA could aid in OA treatment through a direct or indirect regulation of SDF-1 and MMP-3, 9 and 13 levels. This investigated indicated that SDF-1 played a key role in the pathological process of cartilage degeneration of $\mathrm{OA}$ and the research into the intervention on the signal pathway of SDF-1/CXCR4 is expected to find new methods for prevention and treatment of OA.

To sum up, in the treatment of knee OA, the intraarticular injection of HA could effectively reduce the level of SDF-1, MMP-3, MMP-9 and MMP-1 in the synovial fluid and inhibit inflammatory reaction. But it should be used with great caution for treatment of patients with moderate and severe knee OA involving tibial and femoral joints, the joint effusion graded above II or bone contusion.

\section{REFERENCES}

1. Xue Y, Zhang R, Deng Y. A preliminary examination of the diagnostic value of deep learning in hip osteoarthritis. Plos One 2017;12:e0178992.

2. Fouda N, Abd-Elaziz H, Fouda EM. Assessment of subclinical carotid atherosclerosis in patients with primary osteoarthritis: correlation with disease severity and insulin resistance. Egyptian Rheumatol 2014;36:85-91.

3. Jevsevar DS. Treatment of osteoarthritis of the knee: evidencebased guideline. J Am Acad Orthop Surg 2013;21:571-6.

4. Trigkilidas D, Anand A. The effectiveness of hyaluronic acid intra-articular injections in managing osteoarthritic knee pain. Ann R Coll Surg Engl 2013;95:545-51.
5. Trojian TH, ConcoffAL, Joy SM, Hatzenbuehler JR, Saulsberry WJ, Coleman CI. AMSSM scientific statement concerning viscosupplementation injections for knee osteoarthritis: Importance for individual patient outcomes. Br J Sports Med 2016;50:84-92.

6. Askari AT, Unzek S, Popovic ZB. Effect of stromal-cellderived factor 1 on stem-cell homing and tissue regeneration in ischaemic cardiomyopathy. Lancet 2003;362:697-703.

7. Hinman RS, Lentzos J, Vicenzino B, Crossley KM. Is patellofemoral osteoarthritis common in middle-aged people with chronic patellofemoral pain. Arthr care Res 2014;66:12527.

8. McAlindon TE, Bannuru R, Sullivan MC, Arden NK, Berenbaum F, Bierma-Zeinstra SM, et al. OARSI guidelines for the non-surgical management of knee osteoarthritis. Osteoarthritis Cartil 2014;22:363-88.

9. Tubach F, Ravaud P, Baron G, Falissard B, Logeart I, Bellamy $\mathrm{N}$, et al. Evaluation of clinically relevant changes in patient reported outcomes in knee and hip osteoarthritis: the minimal clinically important improvement. Annals Rheumat Dis 2005;64:29-33.

10. Bandak E, Boesen M, Bliddal H, Riis RG, Gudbergsen H, Henriksen M. Associations between muscle perfusion and symptoms in knee osteoarthritis: a cross sectional study. Osteoarthritis Cartil 2015;23:1721-7.

11. Jo DY, Rafii S, Hamada T, Moore MA. Chemotaxis of primitive hematopoietic cells in response to stromal cellderived factor-1. J Clin Invest 2000;105:101-11.

12. Kanbe K, Takemura T, Takeuchi K, Chen Q, Takagishi K, Inoue K. Synovectomy reduces stromal-cell-derived factor-1 (SDF-1) which is involved in the destruction of cartilage in osteoarthritis and rheumatoid arthritis. J Bone Joint Surg 2004;86:296-300.

13. Wei L, Sun X, Kanbe K, Wang Z, Sun C, Terek R, et al. Chondrocyte death induced by pathological concentration of chemokine stromal cell-derived factor-1. J Rheumatol 2006;33:1818-26.

14. Tabata M, Murata E, Ueda K, Kato-Kogoe N, Kuroda Y, Hirose M. Effects of TrkA inhibitory peptide on cancer-induced pain in a mouse melanoma model. J Anaesth 2012;26:545-51.

15. Grecomoro G, La FS, Francavilla G. Rheologic changes in the synovial fluid of patients with gonarthritis induced by intraarticular infiltration of hyaluronic acid. Int J Tissue React 2001;23:67-71.

This is an open access article distributed under the terms of the Creative Commons Attribution-NonCommercial-ShareAlike 3.0 License, which allows others to remix, tweak, and build upon the work non-commercially, as long as the author is credited and the new creations are licensed under the identical terms

This article was originally published in a special issue, "Recent Trends in Biomedical Research" Indian J Pharm Sci 2020:82(1)spl issue1; XX-XX 